EPJ Web of Conferences 38, 17002 (2012)

DOI: $10.1051 /$ epjconf/20123817002

(c) Owned by the authors, published by EDP Sciences, 2012

\title{
Effects of phonon-phonon coupling on properties of pygmy resonance in ${ }^{124-132} \mathrm{Sn}$
}

\author{
N. N. Arsenyev ${ }^{1, a}$, A. P. Severyukhin ${ }^{1}$, V. V. Voronov ${ }^{1}$, and Nguyen Van Giai ${ }^{2}$ \\ 1 Bogoliubov Laboratory of Theoretical Physics, Joint Institute for Nuclear Research, 141980 Dubna, Moscow region, \\ Russia \\ 2 Institut de Physique Nucléaire, CNRS-IN2P3, Université Paris-Sud, F-91406 Orsay Cedex, France
}

\begin{abstract}
Starting from an effective Skyrme interaction we study effects of phonon-phonon coupling on the low-energy electric dipole response in ${ }^{124-132} \mathrm{Sn}$. The QRPA calculations are performed within a finite rank separable approximation. The inclusion of two-phonon configurations gives a considerable contribution to low-lying strength. Comparison with available experimental data shows a reasonable agreement for the low-energy E1 strength distribution.
\end{abstract}

\section{Introduction}

Exotic nuclear collective excitations, like the pygmy dipole resonance, represent a subject of intense investigations during last decades, see for example [1-4]. The structure and dynamics of low-energy dipole strength, also referred to as pygmy dipole resonance (PDR), has extensively been investigated using a variety of theoretical approaches and models [5]. Recent studies have made use of the HartreeFock (HF) plus random phase approximation (RPA) [6], the Hartree-Fock-Bogoliubov (HFB) model plus quasiparticle RPA (QRPA) [7,8], QRPA plus phonon coupling [9], the quasiparticle phonon model (QPM) including complex configurations [1,10], the relativistic RPA [11] and QRPA [12]. Also the quasiparticle time blocking approximation (QTBA) has been used either in a non-relativistic framework [8] or with relativistic Lagrangians (RQTBA) [13, 14].

One of the successful tools for describing the PDR is the QRPA with the self-consistent mean-field derived from Skyrme effective nucleon-nucleon interactions. Such an approach describes the properties of the low-lying states less accurately than more phenomenological ones, but the results are in a reasonable agreement with experimental data (Ref. [5] and references therein). Due to the anharmonicity of vibrations there is a coupling between one-phonon and more complex states $[15,16]$. The main difficulty is that the complexity of calculations beyond standard QRPA increases rapidly with the size of the configuration space, so one has to work within limited spaces. Using a finite rank separable approximation suggested in [17-19] for the residual interaction resulting from Skyrme forces one can overcome this difficulty. In this paper we study the properties of the low-lying and high-lying electric dipole strength in the neutron-rich $\mathrm{Sn}$ isotopes. The couplings between one- and two-phonon components in the wave functions of excited states are taken into account.

\footnotetext{
a e-mail: arsenev@theor.jinr.ru
}

\section{The method}

The calculations are performed by using the SLy4 [20] interaction in the particle-hole $(\mathrm{p}-\mathrm{h})$ channel and a densitydependent zero-range interaction in the particle-particle ( $\mathrm{p}$ p) channel. Spherical symmetry is assumed for the HF ground states. The strength of the surface-peaked zero-range pairing force is taken equal to $940 \mathrm{MeV} \mathrm{fm}^{3}$ in connection with the soft cutoff at $10 \mathrm{MeV}$ above the Fermi energy as introduced in Ref. [18]. This value of the pairing strength is fitted to reproduce the experimental pairing energies for both protons and neutrons.

The residual interaction in the $\mathrm{p}$-h channel $V_{\mathrm{res}}^{\mathrm{ph}}$ and in the p-p channel $V_{\text {res }}^{\mathrm{pp}}$ can be obtained as the second derivative of the energy density functional with respect to the particle density $\rho$ and the pair density $\tilde{\rho}$, respectively. Following Ref. [17] we simplify $V_{\text {res }}^{\mathrm{ph}}$ by approximating it by its Landau-Migdal form. Moreover we neglect the $l=1$ Landau parameters (Landau parameters with $l>1$ are equal to zero in the case of Skyrme interactions). In this work we study only normal parity states and one can neglect the spin-spin terms since they play a minor role. The two-body Coulomb and spin-orbit residual interactions are also dropped. The expressions for $F_{0}^{\mathrm{ph}}, F_{0}^{\prime} \mathrm{ph}$ and $F_{0}^{\mathrm{pp}}, F_{0}^{\prime \mathrm{pp}}$ can be found in Ref. [21] and in Ref. [18], respectively. The Landau parameters $F_{0}, G_{0}, F_{0}^{\prime}, G_{0}^{\prime}$ expressed in terms of the Skyrme force parameters [21] depend on $k_{\mathrm{F}}$. As is pointed out in our previous works $[17,18]$ one needs to adopt some effective value for $k_{\mathrm{F}}$ to give an accurate representation of the original p-h Skyrme interaction. For the present calculations we use the nuclear matter value for $k_{\mathrm{F}}$.

In describing the giant dipole resonance (GDR) we should exclude the spurious state due to the center-of-mass motion of the nucleus. The spurious state admixture can be present as a component in each of the wave functions of the excited states. The spurious $1^{-}$state is excluded from the excitation spectra by introducing the effective neutron $q_{\mathrm{n}}=-Z / A$ and proton $q_{\mathrm{p}}=N / A$ charges [15]. An alternative way of eliminating the spurious state is to orthogonalize it with respect to all physical states [22]. At was shown [6] that eliminating the spurious state by means of effective 
Table 1. Values of the centroid energy and width calculated within the QRPA or QRPA plus phonon-phonon coupling (2PH) in comparison with the experimental values (Expt.) taken from Ref. [2]. The chosen energy interval is $11-20 \mathrm{MeV}$.

\begin{tabular}{llllllll}
\hline \multirow{2}{*}{ Nucleus } & \multicolumn{2}{l}{$\bar{E}(\mathrm{MeV})$} & & & \multicolumn{3}{l}{$\Gamma(\mathrm{MeV})$} \\
\cline { 2 - 3 } \cline { 6 - 7 } & QRPA & 2 PH & Expt. & & QRPA & 2PH & Expt. \\
\hline${ }^{124} \mathrm{Sn}$ & 16.4 & 16.3 & 15.3 & & 4.4 & 4.7 & 4.8 \\
${ }^{126} \mathrm{Sn}$ & 16.2 & 16.2 & & & 4.4 & 4.7 & \\
${ }^{128} \mathrm{Sn}$ & 16.1 & 16.0 & & & 4.7 & 4.7 & \\
${ }^{130} \mathrm{Sn}$ & 15.8 & 15.7 & $15.9(5)$ & & 4.8 & 4.8 & $4.8(1.7)$ \\
${ }^{132} \mathrm{Sn}$ & 15.5 & 15.4 & $16.1(7)$ & & 4.9 & 5.0 & $4.7(2.1)$ \\
\hline
\end{tabular}

charges or orthogonalizing it to all physical states lead to very similar results.

We take into account the coupling between the oneand two-phonon components in the wave functions of excited states. Thus, in the simplest case one can write the wave functions of excited states as [16]

$$
\begin{array}{r}
\Psi_{v}(J M)=\left(\sum_{i} R_{i}(J v) Q_{J M i}^{+}\right. \\
\left.+\sum_{\lambda_{1} i_{1} \lambda_{2} i_{2}} P_{\lambda_{2} i_{2}}^{\lambda_{1} i_{1}}(J v)\left[Q_{\lambda_{1} \mu_{1} i_{1}}^{+} Q_{\lambda_{2} \mu_{2} i_{2}}^{+}\right]_{J M}\right)|0\rangle .
\end{array}
$$

where $|0\rangle$ is the phonon vacuum, $Q_{\lambda \mu i}^{+}|0\rangle$ is the phonon creation operator and $v$ labels the excited states. The coefficients $R_{i}(J v), P_{\lambda_{2} i_{2}}^{\lambda_{1} i_{1}}(J v)$ and energies of the excited states $E_{v}$ are determined by solving the corresponding secular equation of Ref. [19].

The two-phonon configurations of the wave function (1) are constructed from natural-parity phonons with multipolarities $\lambda=1,2,3,4,5$. All dipole excitations with energies below $35 \mathrm{MeV}$ and 15 most collective phonons of $\lambda=2,3,4,5$ multipolarity are included in the wave function (1). It is found that extending the model space for onephonon configurations does not change much the calculated energies and transition probabilities.

\section{Results and discussion}

In figure 1 the calculated dipole spectra for ${ }^{130} \mathrm{Sn}$ are shown. The dipole strength function up to $26 \mathrm{MeV}$ is shown in the right part of the figure. The left panel shows the low-lying parts of the corresponding spectrum (below $12 \mathrm{MeV}$ ). Figure 1 (a) displays the experimental $B(E 1)$ distribution. The black circles with error bars are the experimental data. In order to quantify this resonance-like structure the data are fitted with a Lorentzian distribution [2,3]. Calculations within QRPA are shown in figure 1(b), and the QRPA plus phonon-phonon coupling $(2 \mathrm{PH})$ results are presented in figure 1(c). In the figure, the calculated $E 1$ strength distributions are folded out with a Lorentzian distribution of $1 \mathrm{MeV}$ width. The general shapes of the GDR obtained in the $2 \mathrm{PH}$ are rather close to those observed in experiments. This demonstrates the quality of a description within $2 \mathrm{PH}$ in comparison with QRPA. We conclude that the main mechanisms of the GDR formation in ${ }^{130} \mathrm{Sn}$ are taken into account correctly and consistently in the $2 \mathrm{PH}$ approach.

Let us discuss results for the low-energy $E 1$ strength in more detail (see the left part of figure 1). For the lowlying part of the dipole spectrum, the QRPA calculations

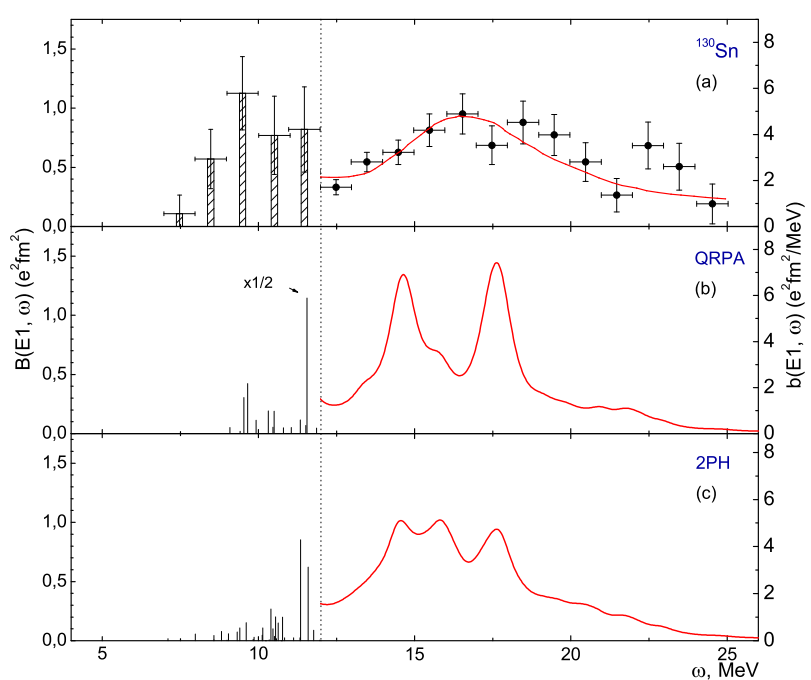

Fig. 1. $B(E 1)$ strength distributions in ${ }^{130} \mathrm{Sn}$. (a) Experimental data [2,3]; (b) QRPA results; (c) QRPA plus phonon-phonon coupling results.

Table 2. Mean energies $\bar{E}$ and summed $B(E 1)$ values for the lowenergy dipole states in the excitation energy range below $11 \mathrm{MeV}$. The experimental values (Expt.) are taken from Refs. [2-4]. To compare with experimental data in ${ }^{124} \mathrm{Sn}$ we choose the energy interval $E \leq 10 \mathrm{MeV}$ [4].

\begin{tabular}{llllllll}
\hline \multirow{2}{*}{ Nucleus } & \multicolumn{2}{l}{$\bar{E}(\mathrm{MeV})$} & & & \multicolumn{3}{c}{$\sum B(E 1)\left(\mathrm{e}^{2} \mathrm{fm}^{2}\right)$} \\
\cline { 2 - 3 } \cline { 6 - 8 } & QRPA & $2 \mathrm{PH}$ & Expt. & & QRPA & 2PH & Expt. \\
\hline${ }^{124} \mathrm{Sn}$ & 9.7 & 9.1 & 6.97 & & 0.86 & 0.59 & $0.379(45)$ \\
${ }^{126} \mathrm{Sn}$ & 10.1 & 10.0 & & & 1.82 & 1.86 & \\
${ }^{128} \mathrm{Sn}$ & 10.0 & 10.0 & & & 1.63 & 1.78 & \\
${ }^{130} \mathrm{Sn}$ & 10.0 & 10.0 & $10.1(7)$ & & 1.40 & 1.80 & $2.4(7)$ \\
${ }^{132} \mathrm{Sn}$ & 9.9 & 9.9 & $9.8(7)$ & & 1.27 & 1.42 & $1.3(8)$ \\
\hline
\end{tabular}

predict two rather pronounced states around 9.5 MeV. The neutron transition densities of these levels dominate outside the nuclear surface. That corresponds to the vibrations of a neutron skin against a proton-neutron core. One can see from figure 1(b) that the next fairly collective state is around $11.6 \mathrm{MeV}$. Using the analysis of the transition densities we found that states above this energy have an intermediate behavior. Increasing further the excitation energy we observe the low-energy GDR tail. The corresponding $2 \mathrm{PH}$ strength distributions in figure 1(c) show many states with comparable strength in the energy region below 11 $\mathrm{MeV}$. The transition densities of these states, originating the fragmentation of the QRPA pygmy mode, have a behavior which is very similar to the behavior of the initial QRPA state. However, we can state that the low-lying transition densities are also dominated by the neutron contribution outside the nuclear surface. Strictly speaking, a correct comparison of the integrated pygmy dipole strength with experimental data in this region is still an open problem, because of loss of experimental sensitivity $[2,3]$.

Now we consider the integral characteristics of the GDR. The GDR calculated characteristics are displayed in Table 1. They are compared with the experimental data [2]. The centroid energy $\bar{E}$ and the spreading width $\Gamma$ are given by the following expressions:

$$
\bar{E}=\frac{m_{1}}{m_{0}}, \quad \Gamma=2.35 \sqrt{\frac{m_{2}}{m_{0}}-\left(\frac{m_{1}}{m_{0}}\right)^{2}},
$$


where $m_{k}=\sum B(E 1) E^{k}$ are the energy-weighted moments. The integral characteristics of $E 1$ strength function in these nuclei have been calculated for the energy interval 11-20 $\mathrm{MeV}$, which exhausts between 100 and $110 \%$ of the Thomas-Reiche-Kuhn energy-weighted sum rules (14.8NZ/A $\mathrm{MeV} \mathrm{e} \mathrm{fm}^{2}$ ).

First of all we checked our approach for ${ }^{124} \mathrm{Sn}$. We obtained a reasonable agreement with available experimental data [2] (see Table 1). In ${ }^{124} \mathrm{Sn}$ we find that $\bar{E}=16.4$ $\mathrm{MeV}$ for the QRPA and $\bar{E}=16.3 \mathrm{MeV}$ for the $2 \mathrm{PH}$, even though the average energy of the giant dipole resonance is by $1 \mathrm{MeV}$ higher than its experimental value. This is in agreement with the RRPA calculations [11] where the centroid energy for GDR is located at $15.86 \mathrm{MeV}$ in the interval 10-25 MeV. For comparison, the QTBA [8] and RQTBA [14] calculations give $15.3 \mathrm{MeV}$ and $15.05 \mathrm{MeV}$, respectively. We note that in the QTBA the $0-30 \mathrm{MeV}$ summation interval is used while in the RQTBA this interval is $10-22.5 \mathrm{MeV}$. One can see from Table 1 that the inclusion of the two-phonon terms results in an increase of the resonance width from 4.4 to $4.7 \mathrm{MeV}$. This value is in agreement with the QTBA calculations $(4.6 \mathrm{MeV})$ [8].

The QRPA results for the neutron-rich ${ }^{130} \mathrm{Sn}$ are presented in figure 1(b). The centroid value of the GDR is 15.8 $\mathrm{MeV}$. The results of our calculations for the $E 1$ resonance taking into account the two-phonon terms are shown in figure 1(c). Here, the GDR centroid energy is $15.7 \mathrm{MeV}$, that is in agreement with the theoretical work using the RRPA [11] $(\bar{E}=15.78 \mathrm{MeV}$ in the $10-25 \mathrm{MeV}$ interval). The RQRPA and RQTBA results are very similar $(\bar{E}=15.13$ $\mathrm{MeV}$ for RQRPA and $\bar{E}=14.66 \mathrm{MeV}$ for RQTBA) in the interval 10-22.5 MeV [13]. For the GDR width we obtain $\Gamma=4.8 \mathrm{MeV}$ that can be compared with the other theoretical values $3.49 \mathrm{MeV}$ in the RQRPA and $4.74 \mathrm{MeV}$ in the RQTBA. The experimental value of the width is equal to 4.8(1.7) $\mathrm{MeV}$ [8].

For ${ }^{132} \mathrm{Sn}$, values calculated by QRPA and $2 \mathrm{PH}$ for the GDR energy centroid are $\bar{E}=15.5 \mathrm{MeV}$ and $\bar{E}=15.4$ $\mathrm{MeV}$, respectively. The experimental value of the GDR width for ${ }^{132} \mathrm{Sn}$ is $4.7(2.1) \mathrm{MeV}$ [2] and this is in agreement with our results. The inclusion of the two-phonon terms gives a small increase of the resonance width from 4.9 to $5.0 \mathrm{MeV}$. Our results for ${ }^{132} \mathrm{Sn}$ are qualitatively similar to calculations in Ref. [9] where the Skyrme interaction SIII has been used. The peak energy and width with the phonon coupling effects are $15.5 \mathrm{MeV}$ and $5.8 \mathrm{MeV}$, respectively. It is worth to note the GDR centroid energy in the interval $8-25 \mathrm{MeV}$ is $14.7 \mathrm{MeV}$ in the RRPA and it is 14.4 $\mathrm{MeV}$ in the RRPA with the inclusion of particle-phonon coupling [12]. The inclusion of particle-phonon coupling in the RRPA calculation results in an increase of the resonance width from 3.3 to $4.0 \mathrm{MeV}$.

One can see that the agreement with the experimental data for ${ }^{124} \mathrm{Sn}$ and ${ }^{130,132} \mathrm{Sn}$ is good. This gives us confidence to use the model for a prediction of the integral characteristics of the GDR in ${ }^{126,128} \mathrm{Sn}$.

In the case ${ }^{126} \mathrm{Sn}$, the centroid energy for the $2 \mathrm{PH}$ case has the same value as for the QRPA calculation 16.2 MeV. One can see that for ${ }^{128} \mathrm{Sn}$ the centroid energy with $2 \mathrm{PH}$ is $16.0 \mathrm{MeV}$, to be compared with the value $16.1 \mathrm{MeV}$ of QRPA. The values of energy centroids for ${ }^{126} \mathrm{Sn}$ and ${ }^{128} \mathrm{Sn}$ are rather close to the empirical systematics $31.2 A^{-1 / 3}+$
$20.6 A^{-1 / 6}$ [23]. The empirical estimations $120 A^{-2 / 3}$ [24] for the GDR widths are reproduced well in both cases.

As it is seen from the Table 1 our calculations show a slight lowering of the GDR peak energy and an increase of the GDR width when passing from ${ }^{124} \mathrm{Sn}$ to ${ }^{132} \mathrm{Sn}$. These results are in an agreement with the calculations by Litvinova [13,14] and Piekarewicz [11]. The role of anharmonic effects, which mainly determine the value of the GDR width, is decreasing when passing from non-magic to magic nuclei.

Finally, let us discuss the low-energy $E 1$ strength. In contrast to the GDR, the effects of phonon-phonon coupling is supposed to be more serious. The integral characteristics of the strength distribution of low-energy dipole states before $11 \mathrm{MeV}$ are given in Table 2. Note that to compare with experimental data in ${ }^{124} \mathrm{Sn}$ we choose the energy interval $E \leq 10 \mathrm{MeV}$ [4].

In the case of ${ }^{124} \mathrm{Sn}$, the QRPA mean energy is equal to $9.7 \mathrm{MeV}$ and taking into account phonon-phonon coupling gives rise to a decrease of the PDR energy by 0.6 $\mathrm{MeV}$, while experiment gives $\bar{E}=6.97 \mathrm{MeV}$ [4]. For comparison, the QTBA [8] and RQTBA [14] calculations give $8.7 \mathrm{MeV}$ and $8.15 \mathrm{MeV}$, respectively. The experimental data for the 4-10 MeV interval give for the integrated PDR strength a value $\sum B(E 1)=0.379(45) \mathrm{e}^{2} \mathrm{fm}^{2}$, while our calculations give values 0.86 and $0.59 \mathrm{e}^{2} \mathrm{fm}^{2}$ within the QRPA and 2PH, respectively. The calculated total QPM [10] dipole strength in the PDR energy range $E=5.7-7.2$ $\mathrm{MeV}$ is $0.324 \mathrm{e}^{2} \mathrm{fm}^{2}$. This is rather close to the experimentally deduced strength. On the other hand, the QTBA [8] and RQTBA [14] results are very similar for the interval below $10 \mathrm{MeV}\left(\sum B(E 1)=3.0 \mathrm{e}^{2} \mathrm{fm}^{2}\right.$ within the QTBA and $\sum B(E 1)=3.2 \mathrm{e}^{2} \mathrm{fm}^{2}$ within the RQTBA).

Values predicted by QRPA and 2PH for the PDR energy centroid in ${ }^{126} \mathrm{Sn}$ are $\bar{E}=10.1 \mathrm{MeV}$ and $\bar{E}=10.0$ $\mathrm{MeV}$, respectively. The inclusion of the two-phonon terms results in a slight increase of the summed $B(E 1)$ from 1.82 to $1.86 \mathrm{e}^{2} \mathrm{fm}^{2}$. In the case of ${ }^{128} \mathrm{Sn}$, the calculated summed $B(E 1)$ strength in the energy range below $11 \mathrm{MeV}$ amounts to $1.63 \mathrm{e}^{2} \mathrm{fm}^{2}$ (in the QRPA) and $1.78 \mathrm{e}^{2} \mathrm{fm}^{2}$ (in the $2 \mathrm{PH}$ ). The centroid energy is $10.0 \mathrm{MeV}$ in both cases.

Our calculations give for ${ }^{130} \mathrm{Sn}$ a total dipole strength $\sum B(E 1)=1.40 \mathrm{e}^{2} \mathrm{fm}^{2}$ for the QRPA and $\sum B(E 1)=1.80$ $\mathrm{e}^{2} \mathrm{fm}^{2}$ for the $2 \mathrm{PH}$. The summation is performed over the dipole states below $11 \mathrm{MeV}$. The experimental value is $\sum B(E 1)=2.4(7) \mathrm{e}^{2} \mathrm{fm}^{2}$ [3]. On the other hand, the corresponding mean energy of the PDR is $10 \mathrm{MeV}$ (it is reproduced in both cases). It may be compared with the experimental value 10.1(7) MeV. The RQRPA and RQTBA results in the interval below $10 \mathrm{MeV}$ [13] are very similar: $\sum B(E 1)=4.04 \mathrm{e}^{2} \mathrm{fm}^{2}$ (RQRPA) and $\sum B(E 1)=3.44$ $\mathrm{e}^{2} \mathrm{fm}^{2}$ (RQTBA). The PDR centroid in the RQTBA is equal to $7.5 \mathrm{MeV}$. For comparison, the RRPA calculations give $\bar{E}=7.91 \mathrm{MeV}$ in the same interval [11]. As one can see from figure 1 the inclusion of the two-phonon terms results in an essential increase of the PDR width $-\Gamma_{P D R}$. For the QRPA case we get $\Gamma_{P D R}=1.0 \mathrm{MeV}$ that can be compared with the value $\Gamma_{P D R}=1.8 \mathrm{MeV}$ when one takes into account the phonon-phonon coupling. Only an upper experimental limit for the PDR width could be deduced because of the finite energy resolution in experiment on Coulomb dissociation [2]. This upper limit for the PDR widths is $\Gamma_{P D R}<3.4 \mathrm{MeV}$. 
Now we discuss the low-lying dipole response in ${ }^{132} \mathrm{Sn}$ for the energy interval below $11 \mathrm{MeV}$. In RPA calculations the mean energy is $9.9 \mathrm{MeV}$. Taking into account phononphonon coupling gives the same value for the PDR energy, while experiment gives $\bar{E}=9.8(7) \mathrm{MeV}$ [2]. We notice that the inclusion of phonon-phonon coupling leads to an increase of the $\sum B(E 1)$ values from 1.27 to $1.42 \mathrm{e}^{2} \mathrm{fm}^{2}$. In the QTBA calculations [8] the centroid energy of PDR is located at $8.9 \mathrm{MeV}$ for the energy interval below $10 \mathrm{MeV}$. This value is by $0.9 \mathrm{MeV}$ lower than the experimental one. For comparison, the RQTBA [14] calculations give 7.3 $\mathrm{MeV}$ for the same energy interval. Our calculation shows that the inclusion of the two-phonon terms increases the resonance width from 1.2 to $2.0 \mathrm{MeV}$. The upper limit for the PDR width is $\Gamma_{P D R}<2.5 \mathrm{MeV}$ [2].

Table 2 demonstrates a distinct difference between characteristics of the low-lying integral strength in ${ }^{124-132} \mathrm{Sn}$. Namely, the contribution of phonon-phonon interaction to $\sum B(E 1)$ is small for nuclei in ${ }^{126,128,130,132} \mathrm{Sn}$, while it is rather important in the ${ }^{124} \mathrm{Sn}$. Moreover, for the nucleus such as ${ }^{124} \mathrm{Sn}$ the PDR is almost completely determined by complex configurations in the energy interval below 10 $\mathrm{MeV}$.

The QRPA predicts a monotonic decrease of the PDR centroid with mass number. The total PDR strength decreases in going to the heavier tin isotopes, up to ${ }^{132} \mathrm{Sn}$. Other QRPA calculations with Skyrme forces [7] give similar tendencies for the summed $B(E 1)$ values, however, our $\sum B(E 1)$ values are somewhat smaller. The influence of coupling between one- and two- phonon terms in the wave functions (1) only leads to the fragmentation of $E 1$ strength. On the other hand, as one can see from figure 1 the inclusion of the two-phonon terms results in an essential increasing of the PDR width. Also from the Table 2 one can see that dipole strength is transferred from the GDR region to the low-energy region. In summary, our $\bar{E}$ and $\sum B(E 1)$ values are in good agreement with the experimental values, except for ${ }^{124} \mathrm{Sn}$.

\section{Summary}

Starting from the Skyrme mean-field calculations, the properties of the electric dipole strength in tin isotopes are studied by taking into account the coupling between one- and two-phonons terms in the wave functions of excited states. The finite rank separable approach for the QRPA calculations enables one to reduce considerably the dimensions of the matrices that must be inverted to perform nuclear structure calculations in very large configuration spaces.

Neutron excess effects on the PDR excitation energy and transition strength have been investigated for ${ }^{124-132} \mathrm{Sn}$. We find that the centroid energy and summed strength decreases when increasing the neutron number. The inclusion of the two-phonon configurations results in an increase of the widths of the PDR and GDR. This is in agreement with available experimental data. For ${ }^{126,128} \mathrm{Sn}$ the properties of the PDR are predicted.

\section{Acknowledgments}

A.N.N., A.P.S. and V.V.V. thank the hospitality of IPNOrsay where a part of this work was done. The authors are thankful to V. Yu. Pomonarev and E. Khan for many fruitful and stimulating discussions concerning various aspect of this work. This work was partly supported by the IN2P3-JINR agreement No. 11-87 and the RFBR No. 1102-91054.

\section{References}

1. K. Govaert, et al., Phys. Rev. C 57, 2229 (1998)

2. P. Adrich, et al., Phys. Rev. Lett. 95, 132501 (2005)

3. A. Klimkiewicz, et al., Phys. Rev. C 76, 051603R (2007)

4. B. Özel, et al., Nucl. Phys. A 788, 385c (2007)

5. N. Paar, et al., Rep. Prog. Phys. 70, 691 (2007)

6. N.N. Arsenyev and A.P. Severyukhin, Phys. Part. Nucl. Lett., 7, 112 (2010)

7. D. Tarpanov, et al., Phys. Atom. Nucl. 70, 1402 (2007)

8. A. Avdeenkov, et al., Phys. Rev. C 83, 064316 (2011)

9. D. Sarchi, P.F. Bortignon, G. Colò, Phys. Lett. B 601, 27 (2004)

10. N. Tsoneva and H. Lenske, Phys. Rev. C 77, 024321 (2008)

11. J. Piekarewicz, Phys. Rev. C 73, 044325 (2006).

12. E. Litvinova, P. Ring, V. Tselyaev, Phys. Rev. C 75, 064308 (2007)

13. E. Litvinova, P. Ring, V. Tselyaev, Phys. Rev. C 78, 014312 (2008)

14. E. Litvinova, et al., Phys. Rev. C 79, 054312 (2009)

15. A. Bohr and B. Mottelson, Nuclear Structure, vol. 2: Nuclear Deformations (Benjamin, New York, 1975)

16. V.G. Soloviev, Theory of Atomic Nuclei: Quasiparticles and Phonons (Institute of Physics, Bristol and Philadelphia, 1992)

17. Nguyen Van Giai, Ch. Stoyanov, V.V. Voronov, Phys. Rev. C 57, 1204 (1998)

18. A.P. Severyukhin, V.V. Voronov, Nguyen Van Giai, Phys. Rev. C 77, 024322 (2008)

19. A.P. Severyukhin, V.V. Voronov, Nguyen Van Giai, Eur. Phys. J. A 22, 397 (2004)

20. E. Chabanat, et al., Nucl. Phys. A 635, 231 (1998)

21. Nguyen Van Giai and H. Sagawa, Phys. Lett. B 106, 379 (1981)

22. G. Colò, et al., Phys. Lett. B 485, 362 (2000)

23. B.L. Berman and S.C. Fultz, Rev. Mod. Phys. 47, 713 (1975)

24. J. Speth and A. Van der Woude, Rep. Prog. Phys. 44, 719 (1981) 